\title{
Weak decay of uniformly accelerated protons and related processes
}

\author{
Daniel A. T. Vanzella and George E. A. Matsas \\ Instituto de Física Teórica, Universidade Estadual Paulista, Rua Pamplona 145, 01405-900, São Paulo, SP, Brazil
}

(Received 31 January 2000; published 6 December 2000)

\begin{abstract}
We investigate the weak interaction emission of spin-1/2 fermions from accelerated currents. As particular applications, we analyze the decay of uniformly accelerated protons and neutrons, and the neutrinoantineutrino emission from uniformly accelerated electrons. The possible relevance of our results to astrophysics is also discussed.
\end{abstract}

DOI: 10.1103/PhysRevD.63.014010

PACS number(s): 13.30.- a, 04.62.+v, 14.20.Dh, 95.30.Cq

\section{INTRODUCTION}

We investigate the weak interaction emission of spin-1/2 fermions from classical and semiclassical currents. We denote by semiclassical those currents which possess classical trajectories and are endowed with quantized inner energy levels. Our results can be used to investigate a broad class of processes involving accelerated particles provided that they have a well defined world line, as indeed verified in many situations of interest. In the case where the particle is accelerated by a background electromagnetic field, such processes could be fully analyzed quantum mechanically. As a consequence, any recoil effects due to the fermion emission would be automatically taken into account $[1,2]$. For instance, in Ref. [1] a quasiclassical approach to quantum electrodynamics was developed to consider $\gamma$-synchrotron radiation from an electron in a classical background magnetic field. This approach is basically characterized by assuming that the electron motion is quasiclassical. This is always possible as long as the magnetic field is not very strong, namely, $H$ $\ll H_{0}$ and $\gamma \gg 1$, where $H_{0}=4.4 \times 10^{13} \mathrm{G}$ and $\gamma$ is the Lorentz factor for the electron. This quasiclassical approach applied to neutrino-antineutrino emission is analyzed in detail in Sec. 6.1 of Ref. [2].

Although it is hard to take into account the current recoil in our semiclassical approach, the relations here obtained, which agree with the full quantum mechanical treatment in the proper limit ( $\chi \ll 1$, see, e.g., Sec. VI), are easily applicable when the process involves particle decay and the trajectory itself (rather than the underlying dynamical process which generates it) is inferred from the observational data. Explicit results for uniformly accelerated currents are exhibited.

As far as we know, the first ones to call attention to the possibility that noninertial protons may decay were Ginzburg and Syrovatskii [3] but only recently Muller [4] obtained the first estimation for the decay rate associated with the process $p \rightarrow n e^{+} \nu_{e}$ by assuming that all the involved particles are scalars. Here, as a particular application of modeling accelerated particles by semiclassical currents, we perform a comprehensive (inertial-frame) analysis of the inverse $\beta$ decay for uniformly accelerated protons. We show that under certain astrophysical conditions, high-energy protons in strong background magnetic fields should rapidly decay.

The observation of noninertial neutrons is less trivial. Notwithstanding the calculation of the $\beta$ decay rate for ac- celerated neutrons may be of some relevance in situations where they are under the influence of "relatively" strong background gravitational fields and, thus, will also be performed.

Some features of the $\beta$ and inverse $\beta$ decays for uniformly accelerated nucleons will be discussed in terms of the Fulling-Davies-Unruh (FDU) effect. The FDU effect asserts that the Minkowski vacuum corresponds to a thermal bath with respect to uniformly accelerated observers $[5,6]$. It is perhaps remarkable that although inertial observers associate the inverse $\beta$ decay to the channel $p \rightarrow n e^{+} \nu_{e}$, coaccelerated observers associate the same proton decay event to one of the following channels: $p e^{-} \rightarrow n \nu_{e}, p \bar{\nu}_{e} \rightarrow n e^{+}$or $p e^{-} \bar{\nu}_{e} \rightarrow n$, where the absorbed $e^{-}$and $\bar{\nu}_{e}$ are Rindler particles present in the FDU thermal bath "attached" to the proton's frame [7]. The corresponding branching ratios can be also calculated as a function of the proton acceleration.

Under a certain restriction, we can make our semiclassical current to behave as a classical one. This is suitable to investigate neutrino-antineutrino emission from accelerated electrons. This process is of relevance in some astrophysical situations as, e.g., in the cooling of neutron stars. Eventually we compare our results in the proper limit with the ones in the literature obtained by quantizing electrons in a background magnetic field [8-10].

The paper is organized as follows. In Sec. II we introduce the semiclassical currents and discuss how they model particle decays. In Sec. III, we introduce the weak-interaction action and couple our current to a spin-1/2 fermionantifermion field. Afterwards we calculate the differential transition probability for currents following arbitrary world lines. In Sec. IV we use the results obtained in the previous section to explicitly evaluate the fermion emission rate and radiated power for the particular case of a uniformly accelerated current. The next two sections, Secs. V and VI, are dedicated to analyze in detail the decay of uniformly accelerated protons and neutrons, and the neutrino-antineutrino emission from uniformly accelerated electrons, respectively. We also comment on the possible astrophysical relevance of our results. We dedicate Sec. VII for our final discussions. We will use natural units $c=\hbar=k_{B}=1$ throughout this paper unless stated otherwise.

\section{SEMICLASSICAL VECTOR CURRENT}

Let us consider a particle in a four-dimensional Minkowski spacetime covered by the usual inertial coordi- 
nates $(t, \mathbf{x}) \in \mathrm{R}^{4}$. Let $x^{\mu}(\tau)$ be the particle's world line and $\tau$ its proper time. The classical vector current associated with this particle is given by

$$
j^{\mu}(x)=\frac{q u^{\mu}(\tau)}{u^{0}(\tau)} \delta^{3}[\mathbf{x}-\mathbf{x}(\tau)],
$$

where $q$ is a "small" coupling constant and $u^{\mu}(\tau)$ $\equiv d x^{\mu} / d \tau$. The current above is suitable to describe a pointlike classical (i.e., with noinner structure) particle. Eventually it can be used to describe a fermion $f_{1}$ and antifermion $\bar{f}_{2}$ emission from an accelerated particle $p_{1}$ :

$$
p_{1} \rightarrow p_{1} f_{1} \bar{f}_{2}
$$

(e.g., a noninertial electron emitting a neutrino-antineutrino pair). Notwithstanding, this current must be improved in order to allow more general processes of the form

$$
p_{1} \rightarrow p_{2} f_{1} \bar{f}_{2},
$$

where particle $p_{1}$ turns into particle $p_{2}$ with a fermionantifermion pair emission (e.g., decay of an accelerated proton into a neutron with a positron-neutrino emission). This is attained by replacing the real coupling constant $q$ by an operator-valued function (see, e.g., Ref. [11])

$$
\hat{q}(\tau)=e^{i \hat{H}_{0} \tau} \hat{q}_{0} e^{-i \hat{H}_{0} \tau}
$$

This can be regarded as the usual first-quantization procedure, where a classical observable $q$ is replaced by a selfadjoint operator $\hat{q}_{0}$ evolved by the one-parameter group of unitary operators $\hat{\mathrm{U}}(\tau)=e^{-i \hat{H}_{0} \tau}$. Here $\hat{H}_{0}$ is the proper Hamiltonian of the system, i.e.,

$$
\hat{H}_{0}\left|p_{j}\right\rangle=M_{j}\left|p_{j}\right\rangle, \quad j=1,2,
$$

where $\left|p_{1}\right\rangle$ and $\left|p_{2}\right\rangle$ are the energy eigenstates associated with particles $p_{1}$ and $p_{2}$, respectively, and $M_{1}$ and $M_{2}$ are the corresponding rest masses. As a result, the classical current (2.1) is replaced by the semiclassical one

$$
\hat{j}^{\mu}(x)=\frac{\hat{q}(\tau) u^{\mu}(\tau)}{u^{0}(\tau)} \delta^{3}[\mathbf{x}-\mathbf{x}(\tau)] .
$$

Calculating the matrix elements $j_{\left(p_{i} \rightarrow p_{j}\right)}^{\mu} \equiv\left\langle p_{j}\left|\hat{j}^{\mu}\right| p_{i}\right\rangle$ associated with $\hat{j}^{\mu}$, we have

$$
j_{\left(p_{i} \rightarrow p_{j}\right)}^{\mu}=G_{\mathrm{eff}} e^{i\left(M_{j}-M_{i}\right) \tau} \frac{u^{\mu}(\tau)}{u^{0}(\tau)} \delta^{3}[\mathbf{x}-\mathbf{x}(\tau)],
$$

where $G_{\text {eff }} \equiv\left|\left\langle p_{2}\left|\hat{q}_{0}\right| p_{1}\right\rangle\right|$ is the effective coupling constant. Note that we can recover current (2.1) from Eq. (2.6) by making $M_{2}=M_{1}$ and $G_{\text {eff }}=q$.

We will assume that the fermion emission does not change appreciably the four-velocity of $p_{2}$ with respect to $p_{1}$. We will denominate this assumption "no-recoil condition." This is verified as far as the momentum of the emitted fermions (with respect to the inertial frame instantaneously at rest with the current) satisfies $|\widetilde{\mathbf{k}}| \ll M_{1}, M_{2}$. In order to be conservative we will impose $|\widetilde{\mathbf{k}}|<\tilde{\omega} \ll M_{1}, M_{2}$. It will become clear further that the typical energy of the emitted fermions $\tilde{\omega}$ is of the order of the current's proper acceleration $a$. Hence our condition above can be recast in the suitable form $a \ll M_{1}, M_{2}$. Our results should be accurate as far as this condition is verified.

\section{FERMION-ANTIFERMION EMISSION FROM A SEMICLASSICAL CURRENT}

We shall describe the emitted fermions by spinorial fields

$$
\hat{\Psi}(x)=\sum_{\sigma= \pm} \int d^{3} \mathbf{k}\left[\hat{b}_{\mathbf{k} \sigma} \psi_{\mathbf{k} \sigma}^{(+\omega)}(x)+\hat{d}_{\mathbf{k} \sigma}^{\dagger} \psi_{-\mathbf{k}-\sigma}^{(-\omega)}(x)\right]
$$

where $\hat{b}_{\mathbf{k} \sigma}$ and $\hat{d}_{\mathbf{k} \sigma}^{\dagger}$ are annihilation and creation operators of fermions and antifermions, respectively, with threemomentum $\mathbf{k}=\left(k^{x}, k^{y}, k^{z}\right)$ and polarization $\sigma$. We will adopt the notation used in Ref. [7]. Energy $\omega$, momentum k, and mass $m$ are related as usual: $\omega=\sqrt{\mathbf{k}^{2}+m^{2}}>0 . \psi_{\mathbf{k} \sigma}^{(+\omega)}$ and $\psi_{\mathbf{k} \sigma}^{(-\omega)}$ are positive and negative frequency solutions of the Dirac equation $i \gamma^{\mu} \partial_{\mu} \psi_{\mathbf{k} \sigma}^{( \pm \omega)}-m \psi_{\mathbf{k} \sigma}^{( \pm \omega)}=0$. By using the $\gamma^{\mu}$ matrices in the Dirac representation (see, e.g., Ref. [12]), we find

$$
\psi_{\mathbf{k}+\omega)}^{( \pm \omega)}(x) \frac{e^{i(\mp \omega t+\mathbf{k} \cdot \mathbf{x})}}{\sqrt{16 \pi^{3} \omega(\omega \pm m)}}\left(\begin{array}{c}
m \pm \omega \\
0 \\
k^{z} \\
k^{x}+i k^{y}
\end{array}\right)
$$

and

$$
\psi_{\mathbf{k}-}^{( \pm \omega)}(x)=\frac{e^{i(\mp \omega t+\mathbf{k} \cdot \mathbf{x})}}{\sqrt{16 \pi^{3} \omega(\omega \pm m)}}\left(\begin{array}{c}
0 \\
m \pm \omega \\
k^{x}-i k^{y} \\
-k^{z}
\end{array}\right)
$$

We have orthonormalized modes (3.2), (3.3) according to the inner product [11]

$$
\begin{aligned}
\left\langle\psi_{\mathbf{k} \sigma}^{( \pm \omega)}, \psi_{\mathbf{k}^{\prime} \sigma^{\prime}}^{\left( \pm \omega^{\prime}\right)}\right\rangle & \equiv \int_{\Sigma} d \Sigma_{\mu} \bar{\psi}_{\mathbf{k} \sigma}^{( \pm \omega)} \gamma^{\mu} \psi_{\mathbf{k}^{\prime} \sigma^{\prime}}^{\left( \pm \omega^{\prime}\right)} \\
& =\delta^{3}\left(\mathbf{k}-\mathbf{k}^{\prime}\right) \delta_{\sigma \sigma^{\prime}} \delta_{ \pm \omega \pm \omega^{\prime}}
\end{aligned}
$$

where $d \Sigma_{\mu} \equiv n_{\mu} d \Sigma$ with $n^{\mu}$ being a unit vector orthogonal to $\Sigma$ and pointing to the future, and $\Sigma$ is an arbitrary spacelike hypersurface. We have chosen $t=$ const for the hypersurface $\Sigma$. As a consequence, canonical anticommutation relations for fields and conjugate momenta lead to the following simple anticommutation relations for creation and annihilation operators:

$$
\left\{\hat{b}_{\mathbf{k} \sigma}, \hat{b}_{\mathbf{k}^{\prime} \sigma^{\prime}}^{\dagger}\right\}=\left\{\hat{d}_{\mathbf{k} \sigma}, \hat{d}_{\mathbf{k}^{\prime} \sigma^{\prime}}^{\dagger}\right\}=\delta^{3}\left(\mathbf{k}-\mathbf{k}^{\prime}\right) \delta_{\sigma \sigma^{\prime}}
$$


and

$$
\left\{\hat{b}_{\mathbf{k} \sigma}, \hat{b}_{\mathbf{k}^{\prime} \sigma^{\prime}}\right\}=\left\{\hat{d}_{\mathbf{k} \sigma}, \hat{d}_{\mathbf{k}^{\prime} \sigma^{\prime}}\right\}=\left\{\hat{b}_{\mathbf{k} \sigma}, \hat{d}_{\mathbf{k}^{\prime} \sigma^{\prime}}\right\}=\left\{\hat{b}_{\mathbf{k} \sigma}, \hat{d}_{\mathbf{k}^{\prime} \sigma^{\prime}}^{\dagger}\right\}=0 .
$$

Next we minimally couple the spinorial fields $\hat{\Psi}_{1}$ and $\hat{\Psi}_{2}$, associated with the emitted fermions $f_{1}$ and $\bar{f}_{2}$, respectively, to our general current $\hat{j}^{\mu}$ according to the weak-interaction action

$\hat{S}_{I}=\int d^{4} x \hat{j}_{\mu}\left\{\hat{\bar{\Psi}}_{1} \gamma^{\mu}\left(c_{V}-c_{A} \gamma^{5}\right) \hat{\Psi}_{2}+\hat{\bar{\Psi}}_{2} \gamma^{\mu}\left(c_{V}-c_{A} \gamma^{5}\right) \hat{\Psi}_{1}\right\}$,

where $c_{V}$ and $c_{A}$ will be settled further.

The vacuum transition amplitude for process (2.2) at the tree level is given by

$$
\mathcal{A}_{\mathbf{k}_{1} \mathbf{k}_{2}}^{\sigma_{1} \sigma_{2}}=\left\langle p_{2}\left|\otimes\left\langle f_{1 \mathbf{k}_{1} \sigma_{1}}, \overline{f_{2} \mathbf{k}_{2} \sigma_{2}}\left|\hat{S}_{I}\right| 0\right\rangle \otimes\right| p_{1}\right\rangle
$$

Note that the second term inside the parenthesis at the right hand side of Eq. (3.7) vanishes in this case. By using the field decomposition (3.1) in Eq. (3.7), and acting $\hat{S}_{I}$ in Eq. (3.8), we obtain

$$
\mathcal{A}_{\mathbf{k}_{1} \mathbf{k}_{2}}^{\sigma_{1} \sigma_{2}}=\int d^{4} x j_{\mu}^{\left(p_{1} \rightarrow p_{2}\right)} \bar{\psi}_{\mathbf{k}_{1} \sigma_{1}}^{\left(+\omega_{1}\right)} \gamma^{\mu}\left(c_{V}-c_{A} \gamma^{5}\right) \psi_{-\mathbf{k}_{2}-\sigma_{2}}^{\left(-\omega_{2}\right)},
$$

where $j_{\mu}^{\left(p_{i} \rightarrow p_{j}\right)}$ and $\psi_{\mathbf{k}_{j} \sigma_{j}}^{\left( \pm \omega_{j}\right)}$ are obtained from Eqs. (2.6) and (3.2), (3.3), respectively.

By substituting the amplitude (3.9) in the following expression for the differential transition probability

$$
\frac{d \mathcal{P}^{p_{1} \rightarrow p_{2}}}{d^{3} \mathbf{k}_{1} d^{3} \mathbf{k}_{2}}=\sum_{\sigma_{1}= \pm} \sum_{\sigma_{2}= \pm}\left|\mathcal{A}_{\mathbf{k}_{1} \mathbf{k}_{2}}^{\sigma_{1} \sigma_{2}}\right|^{2}
$$

we obtain

$$
\frac{d \mathcal{P}^{p_{1} \rightarrow p_{2}}}{d^{3} \mathbf{k}_{1} d^{3} \mathbf{k}_{2}}=\int d^{4} x \int d^{4} x^{\prime} \mathbf{J}_{\mu \nu}^{\left(p_{1} \rightarrow p_{2}\right)}\left(x, x^{\prime}\right) \mathrm{G}_{\mathbf{k}_{1} \mathbf{k}_{2}}^{\mu \nu}\left(x, x^{\prime}\right),
$$

where

$$
\mathbf{J}_{\mu \nu}^{\left(p_{1} \rightarrow p_{2}\right)}\left(x, x^{\prime}\right) \equiv j_{\mu}^{\left(p_{1} \rightarrow p_{2}\right)}(x) j_{\nu}^{\left(p_{2} \rightarrow p_{1}\right)}\left(x^{\prime}\right),
$$

and

$$
\begin{aligned}
\mathrm{G}_{\mathbf{k}_{1} \mathbf{k}_{2}}^{\mu \nu}\left(x, x^{\prime}\right) \equiv & \sum_{\sigma_{1}= \pm} \sum_{\sigma_{2}= \pm}\left\{\bar{\psi}_{\mathbf{k}_{1} \sigma_{1}}^{\left(+\omega_{1}\right)}(x) \gamma^{\mu}\left(c_{V}-c_{A} \gamma^{5}\right)\right. \\
& \times \psi_{-\mathbf{k}_{2}-\sigma_{2}}^{\left(-\omega_{2}\right)}(x) \bar{\psi}_{-\mathbf{k}_{2}-\sigma_{2}}^{\left(-\omega_{2}\right)}\left(x^{\prime}\right) \\
& \left.\times \gamma^{\nu}\left(c_{V}-c_{A} \gamma^{5}\right) \psi_{\mathbf{k}_{1} \sigma_{1}}^{\left(+\omega_{1}\right)}\left(x^{\prime}\right)\right\}
\end{aligned}
$$

Equation (3.12) can be cast in the form

$$
\begin{aligned}
\mathbf{J}_{\mu \nu}^{\left(p_{1} \rightarrow p_{2}\right)}\left(x, x^{\prime}\right)= & G_{\mathrm{eff}}^{2} \frac{u_{\mu}(\tau) u_{\nu}\left(\tau^{\prime}\right)}{u^{0}(\tau) u^{0}\left(\tau^{\prime}\right)} e^{i \Delta M\left(\tau-\tau^{\prime}\right)} \delta^{3}[\mathbf{x}-\mathbf{x}(\tau)] \\
& \times \delta^{3}\left[\mathbf{x}^{\prime}-\mathbf{x}\left(\tau^{\prime}\right)\right]
\end{aligned}
$$

by using our current (2.5), where $\Delta M \equiv M_{2}-M_{1}$, while Eq. (3.13) is written as

$$
\begin{aligned}
\mathrm{G}_{\mathbf{k}_{1} \mathbf{k}_{2}}^{\mu \nu}\left(x, x^{\prime}\right)= & \operatorname{tr}\left\{\gamma ^ { \mu } ( c _ { V } - c _ { A } \gamma ^ { 5 } ) \sum _ { \sigma _ { 2 } = \pm } \left[\psi_{-\mathbf{k}_{2}-\sigma_{2}}^{\left(-\omega_{2}\right)}(x)\right.\right. \\
& \left.\times \bar{\psi}_{-\mathbf{k}_{2}-\sigma_{2}}^{\left(-\omega_{2}\right)}\left(x^{\prime}\right)\right] \gamma^{\nu}\left(c_{V}-c_{A} \gamma^{5}\right) \\
& \left.\times \sum_{\sigma_{1}= \pm}\left[\psi_{\mathbf{k}_{1} \sigma_{1}}^{\left(+\omega_{1}\right)}\left(x^{\prime}\right) \bar{\psi}_{\mathbf{k}_{1} \sigma_{1}}^{\left(+\omega_{1}\right)}(x)\right]\right\} .
\end{aligned}
$$

The summations that appear in Eq. (3.15) can be calculated by using modes (3.2), (3.3):

$$
\sum_{\sigma= \pm} \psi_{ \pm \mathbf{k} \sigma}^{( \pm \omega)}(x) \bar{\psi}_{ \pm \mathbf{k} \sigma}^{( \pm \omega)}\left(x^{\prime}\right)=\frac{(k \pm m)}{2(2 \pi)^{3} \omega} e^{ \pm i k^{\lambda}\left(x-x^{\prime}\right) \lambda}
$$

where $k^{\lambda}=(\omega, \mathbf{k})$ is the emitted fermion's four momentum and $k=k^{\lambda} \gamma_{\lambda}$. Applying the above expression in Eq. (3.15), and using $\gamma$-matrix trace identities, we obtain

$$
\begin{aligned}
\mathrm{G}_{\mathbf{k}_{1} \mathbf{k}_{2}}^{\mu \nu}\left(x, x^{\prime}\right)= & \frac{e^{i\left(k_{1}+k_{2}\right)^{\lambda}\left(x-x^{\prime}\right)_{\lambda}}}{4(2 \pi)^{6} \omega_{1} \omega_{2}}\left\{\left(c_{V}^{2}+c_{A}^{2}\right) \operatorname{tr}\left[\gamma^{\mu} k_{2} \gamma^{\nu} k_{1}\right]\right. \\
& +2 c_{V} c_{A} \operatorname{tr}\left[\gamma^{5} \gamma^{\mu} k_{2} \gamma^{\nu} k_{1}\right] \\
& \left.-m_{1} m_{2}\left(c_{V}^{2}-c_{A}^{2}\right) \operatorname{tr}\left[\gamma^{\mu} \gamma^{\nu}\right]\right\} \\
= & \frac{e^{i\left(k_{1}+k_{2}\right)^{\lambda}\left(x-x^{\prime}\right)} \lambda}{(2 \pi)^{6} \omega_{1} \omega_{2}}\left\{\left(c_{V}^{2}+c_{A}^{2}\right)\right. \\
& \times\left[2 k_{1}^{(\mu} k_{2}^{\nu)}-\eta^{\mu \nu} k_{1}^{\alpha} k_{2 \alpha}\right]-m_{1} m_{2}\left(c_{V}^{2}-c_{A}^{2}\right) \eta^{\mu \nu} \\
& \left.+2 i c_{V} c_{A} \epsilon^{\mu \nu \alpha \beta} k_{1 \alpha} k_{2 \beta}\right\},
\end{aligned}
$$

where $\epsilon^{\mu \alpha \nu \beta}$ is the totally skew-symmetric Levi-Civita pseudotensor (with $\epsilon^{0123}=-1$ ) and $k_{1}^{(\mu} k_{2}^{\nu)} \equiv\left(k_{1}^{\mu} k_{2}^{\nu}\right.$ $\left.+k_{1}^{\nu} k_{2}^{\mu}\right) / 2$. Letting Eqs. (3.14) and (3.17) into (3.11), we obtain the differential transition probability

$$
\begin{aligned}
\frac{d \mathcal{P}^{p_{1} \rightarrow p_{2}}}{d^{3} \mathbf{k}_{1} d^{3} \mathbf{k}_{2}}= & \frac{G_{\mathrm{eff}}^{2}}{(2 \pi)^{6} \omega_{1} \omega_{2}} \int_{-\infty}^{+\infty} d \tau \int_{-\infty}^{+\infty} d \tau^{\prime} e^{i \Delta M\left(\tau-\tau^{\prime}\right)} \\
& \times e^{i\left(k_{1}+k_{2}\right)^{\lambda}\left[x(\tau)-x\left(\tau^{\prime}\right)\right]_{\lambda}\left\{2 \left[\left(c_{V}^{2}+c_{A}^{2}\right) k_{1}^{(\mu} k_{2}^{\nu)}\right.\right.} \\
& \left.+i c_{V} c_{A} \epsilon^{\mu \nu \alpha \beta} k_{1 \alpha} k_{2 \beta}\right] u_{\mu}(\tau) u_{\nu}\left(\tau^{\prime}\right)-\left[\left(c_{V}^{2}-c_{A}^{2}\right)\right. \\
& \left.\left.\times m_{1} m_{2}+\left(c_{V}^{2}+c_{A}^{2}\right) k_{1}^{\alpha} k_{2 \alpha}\right] u^{\mu}(\tau) u_{\mu}\left(\tau^{\prime}\right)\right\}
\end{aligned}
$$

where we have used that $d \tau=d t / u^{0}$ 


\section{UNIFORMLY ACCELERATED CURRENTS}

The world line of a uniformly accelerated particle with proper acceleration $a$ can be given in the usual Minkowski coordinates $(t, \mathbf{x}) \in \mathrm{R}^{4}$ by

$$
x^{\mu}(\tau)=\left(a^{-1} \sinh a \tau, 0,0, a^{-1} \cosh a \tau\right) .
$$

The corresponding four-velocity is

$$
u^{\mu}(\tau)=(\cosh a \tau, 0,0, \sinh a \tau) .
$$

Let us now define new coordinates

$$
\xi \equiv\left(\tau-\tau^{\prime}\right) / 2 \quad \text { and } s \equiv\left(\tau+\tau^{\prime}\right) / 2 \text {, }
$$

which allows us to rewrite Eq. (3.18) as

$$
\begin{aligned}
\frac{d \mathcal{P}^{p_{1} \rightarrow p_{2}}}{d^{3} \mathbf{k}_{1} d^{3} \mathbf{k}_{2}}= & \frac{2 G_{\mathrm{eff}}^{2}}{(2 \pi)^{6} \omega_{1} \omega_{2}} \int_{-\infty}^{+\infty} d s \int_{-\infty}^{+\infty} d \xi \exp \{2 i[\Delta M \xi \\
& \left.\left.+\left(k_{1}+k_{2}\right)^{\lambda} u_{\lambda}(s) \sinh (a \xi) / a\right]\right\} \\
& \times\left\{2 ( c _ { V } ^ { 2 } + c _ { A } ^ { 2 } ) k _ { 1 } ^ { \mu } k _ { 2 } ^ { \nu } \left[u_{\mu}(s) u_{\nu}(s) \cosh ^{2}(a \xi)\right.\right. \\
& \left.-a^{2} x_{\mu}(s) x_{\nu}(s) \sinh ^{2}(a \xi)\right]-\cosh (2 a \xi) \\
& \times\left[\left(c_{V}^{2}-c_{A}^{2}\right) m_{1} m_{2}+\left(c_{V}^{2}+c_{A}^{2}\right)\left(k_{1}^{\alpha} k_{2 \alpha}\right)\right] \\
& \left.+2 i a c_{V} c_{A} \sinh (2 a \xi) \epsilon_{\mu \nu \alpha \beta} x^{\mu}(s) u^{\nu}(s) k_{1}^{\alpha} k_{2}^{\beta}\right\},
\end{aligned}
$$

where we have used $\left[x(\tau)-x\left(\tau^{\prime}\right)\right]^{\mu}=2 a^{-1} \sinh (a \xi) u^{\mu}(s)$, $u^{\mu}(\tau)=\cosh (a \xi) u^{\mu}(s)+a \sinh (a \xi) x^{\mu}(s)$, $u^{\mu}\left(\tau^{\prime}\right)$ $=\cosh (a \xi) u^{\mu}(s)-a \sinh (a \xi) x^{\mu}(s), \quad$ and $\quad u^{\mu}(\tau) u_{\mu}\left(\tau^{\prime}\right)$ $=\cosh (2 a \xi)$.

In order to decouple the integrals in Eq. (4.4), let us make the following change in the momentum variable:

$$
k^{\mu} \rightarrow \widetilde{k}^{\mu}=(\tilde{\omega}, \widetilde{\mathbf{k}}) \equiv\left[k^{\lambda} u_{\lambda}(s), k^{x}, k^{y},-a k^{\lambda} x_{\lambda}(s)\right] .
$$

Using Eqs. (4.1) and (4.2) we can verify explicitly that the transformation (4.5) corresponds to a boost in the $z$ direction. Indeed, $\widetilde{k}^{\mu}$ are the components of the emitted fermion's fourmomentum in the inertial frame instantaneously at rest with the current at the proper time $s$. Hence the transition probability per proper time $\Gamma^{p_{1} \rightarrow p_{2}} \equiv d \mathcal{P}^{p_{1} \rightarrow p_{2}} / d s$ for process (2.2) can be written from Eq. (4.4) as

$$
\begin{aligned}
\frac{d \Gamma^{p_{1} \rightarrow p_{2}}}{d^{3} \widetilde{\mathbf{k}}_{1} d^{3} \widetilde{\mathbf{k}}_{2}}= & \frac{2 G_{\mathrm{eff}}^{2}}{(2 \pi)^{6} \tilde{\omega}_{1} \tilde{\omega}_{2}} \int_{-\infty}^{+\infty} d \xi \exp \{2 i[\Delta M \xi \\
& \left.\left.+a^{-1} \sinh (a \xi)\left(\tilde{\omega}_{1}+\tilde{\omega}_{2}\right)\right]\right\} \\
& \times\left\{\left(c_{V}^{2}+c_{A}^{2}\right)\left(\tilde{\omega}_{1} \tilde{\omega}_{2}+\widetilde{k}_{1}^{z} \widetilde{k}_{2}^{z}\right)\right. \\
& -2 i c_{V} c_{A} \sinh (2 a \xi)\left(\widetilde{\mathbf{k}}_{1} \times \widetilde{\mathbf{k}}_{2}\right)^{z}+\left[\left(c_{V}^{2}+c_{A}^{2}\right)\right. \\
& \left.\left.\times\left(\widetilde{\mathbf{k}}_{1}^{\perp} \cdot \widetilde{\mathbf{k}}_{2}^{\perp}\right)-\left(c_{V}^{2}-c_{A}^{2}\right) m_{1} m_{2}\right] \cosh (2 a \xi)\right\},
\end{aligned}
$$

where $\widetilde{\mathbf{k}}_{1} \times \tilde{\mathbf{k}}_{2}$ is the usual three-vector product and $\tilde{\mathbf{k}}_{1}^{\perp} \cdot \tilde{\mathbf{k}}_{2}^{\perp}$ $\equiv \widetilde{k}_{1}^{x} \widetilde{k}_{2}^{x}+\widetilde{k}_{1}^{y} \widetilde{k}_{2}^{y}$. In order to integrate Eq. (4.6), it is convenient to use spherical coordinates in the momenta space $(\widetilde{k}$ $\left.\in \mathrm{R}^{+}, \widetilde{\theta} \in[0, \pi], \widetilde{\phi} \in[0,2 \pi)\right)$, where $\widetilde{k}^{x}=\widetilde{k} \sin \widetilde{\theta} \cos \widetilde{\phi}, \quad \widetilde{k}^{y}$ $=\widetilde{k} \sin \widetilde{\theta} \sin \widetilde{\phi}, \widetilde{k}^{z}=\widetilde{k} \cos \widetilde{\theta}$, and the following change of integration variable: $\xi \rightarrow \lambda \equiv e^{a \xi}$. By using expression (3.471.10) of Ref. [13], we obtain

$$
\begin{aligned}
\frac{d \Gamma^{p_{1} \rightarrow p_{2}}}{d^{3} \widetilde{\mathbf{k}}_{1} d^{3} \widetilde{\mathbf{k}}_{2}}= & \frac{4 G_{\mathrm{eff}}^{2} e^{-\pi \Delta M / a}}{(2 \pi)^{6} \tilde{\omega}_{1} \tilde{\omega}_{2} a}\left\{( c _ { V } ^ { 2 } + c _ { A } ^ { 2 } ) \left(\tilde{\omega}_{1} \tilde{\omega}_{2}+\widetilde{k}_{1} \tilde{k}_{2}\right.\right. \\
& \left.\times \cos \widetilde{\theta}_{1} \cos \widetilde{\theta}_{2}\right) K_{2 i \Delta M / a}\left[2\left(\tilde{\omega}_{1}+\tilde{\omega}_{2}\right) / a\right] \\
& +2 c_{V} c_{A} \widetilde{k}_{1} \tilde{k}_{2} \sin \widetilde{\theta}_{1} \sin \widetilde{\theta}_{2} \sin \left(\widetilde{\phi}_{1}-\widetilde{\phi}_{2}\right) \\
& \times \operatorname{Im}\left\{K_{2+2 i \Delta M / a}\left[2\left(\tilde{\omega}_{1}+\tilde{\omega}_{2}\right) / a\right]\right\} \\
& +\left[\left(c_{V}^{2}-c_{A}^{2}\right) m_{1} m_{2}-\left(c_{V}^{2}+c_{A}^{2}\right)\right. \\
& \left.\times \widetilde{k}_{1} \widetilde{k}_{2} \sin \widetilde{\theta}_{1} \sin \widetilde{\theta}_{2} \cos \left(\widetilde{\phi}_{1}-\widetilde{\phi}_{2}\right)\right] \\
& \left.\times \operatorname{Re}\left\{K_{2+2 i \Delta M / a}\left[2\left(\tilde{\omega}_{1}+\tilde{\omega}_{2}\right) / a\right]\right\}\right\}
\end{aligned}
$$

where $\operatorname{Re}\{z\}$ and $\operatorname{Im}\{z\}$ are the real and imaginary parts of a complex number $z$, respectively, and $K_{\nu}(z)$ is the modified Bessel function.

We note that the uncorrelated emission of $f_{1}$ and $\bar{f}_{2}$ is spherically symmetric in the instantaneously comoving frame. This can be seen by tracing out (i.e., integrating) one of the momentum variables in Eq. (4.7),

$$
\begin{aligned}
\frac{d \Gamma^{p_{1} \rightarrow p_{2}}}{d^{3} \widetilde{\mathbf{k}}_{j}}= & \frac{8 G_{\mathrm{eff}}^{2} e^{-\pi \Delta M / a}}{(2 \pi)^{5} \tilde{\omega}_{j} a} \int_{0}^{\infty} d \widetilde{k}_{l} \frac{\tilde{k}_{l}^{2}}{\tilde{\omega}_{l}}\left(\left(c_{V}^{2}+c_{A}^{2}\right) \tilde{\omega}_{1} \tilde{\omega}_{2}\right. \\
& \times K_{2 i \Delta M / a}\left[2\left(\tilde{\omega}_{1}+\tilde{\omega}_{2}\right) / a\right]+\left(c_{V}^{2}-c_{A}^{2}\right) m_{1} m_{2} \\
& \left.\times \operatorname{Re}\left\{K_{2+2 i \Delta M / a}\left[2\left(\tilde{\omega}_{1}+\tilde{\omega}_{2}\right) / a\right]\right\}\right),
\end{aligned}
$$

and noting that this expression is independent of $\left(\widetilde{\theta}_{j}, \widetilde{\phi}_{j}\right)$, where $j, l=1$ and 2 are associated with particles $f_{1}$ and $\bar{f}_{2}$. The energy distribution of emitted particles is given by

$$
\begin{aligned}
\frac{d \Gamma^{p_{1} \rightarrow p_{2}}}{d \tilde{\omega}_{j}}= & \frac{G_{\mathrm{eff}}^{2} e^{-\pi \Delta M / a}}{\pi^{4} a} \sqrt{\tilde{\omega}_{j}^{2}-m_{j}^{2}} \int_{m_{l}}^{\infty} d \tilde{\omega}_{l} \sqrt{\tilde{\omega}_{l}^{2}-m_{l}^{2}} \\
& \times\left(\left(c_{V}^{2}+c_{A}^{2}\right) \tilde{\omega}_{1} \tilde{\omega}_{2} K_{2 i \Delta M / a}\left[2\left(\tilde{\omega}_{1}+\tilde{\omega}_{2}\right) / a\right]\right. \\
& +\left(c_{V}^{2}-c_{A}^{2}\right) m_{1} m_{2} \\
& \left.\times \operatorname{Re}\left\{K_{2+2 i \Delta M / a}\left[2\left(\tilde{\omega}_{1}+\tilde{\omega}_{2}\right) / a\right]\right\}\right) .
\end{aligned}
$$

The total transition rate is given by 


$$
\begin{aligned}
\Gamma^{p_{1} \rightarrow p_{2}=} & \frac{G_{\mathrm{eff}}^{2} e^{-\pi \Delta M / a}}{\pi^{4} a} \int_{m_{1}}^{\infty} d \tilde{\omega}_{1} \int_{m_{2}}^{\infty} d \tilde{\omega}_{2} \sqrt{\tilde{\omega}_{1}^{2}-m_{1}^{2}} \sqrt{\tilde{\omega}_{2}^{2}-m_{2}^{2}} \\
& \times\left(\left(c_{V}^{2}+c_{A}^{2}\right) \tilde{\omega}_{1} \tilde{\omega}_{2} K_{2 i \Delta M / a}\left[2\left(\tilde{\omega}_{1}+\tilde{\omega}_{2}\right) / a\right]\right. \\
& \left.+\left(c_{V}^{2}-c_{A}^{2}\right) m_{1} m_{2} \operatorname{Re}\left\{K_{2+2 i \Delta M / a}\left[2\left(\tilde{\omega}_{1}+\tilde{\omega}_{2}\right) / a\right]\right\}\right)
\end{aligned}
$$

while the emitted power can be estimated by

$$
\begin{aligned}
\mathcal{W}_{j}^{p_{1} \rightarrow p_{2}}= & \frac{G_{\mathrm{eff}}^{2} e^{-\pi \Delta M / a}}{\pi^{4} a} \int_{m_{1}}^{\infty} d \tilde{\omega}_{1} \int_{m_{2}}^{\infty} d \tilde{\omega}_{2} \tilde{\omega}_{j} \\
& \times \sqrt{\tilde{\omega}_{1}^{2}-m_{1}^{2}} \sqrt{\tilde{\omega}_{2}^{2}-m_{2}^{2}}\left(\left(c_{V}^{2}+c_{A}^{2}\right)\right. \\
& \times \tilde{\omega}_{1} \tilde{\omega}_{2} K_{2 i \Delta M / a}\left[2\left(\tilde{\omega}_{1}+\tilde{\omega}_{2}\right) / a\right]+\left(c_{V}^{2}-c_{A}^{2}\right) \\
& \left.\times m_{1} m_{2} \operatorname{Re}\left\{K_{2+2 i \Delta M / a}\left[2\left(\tilde{\omega}_{1}+\tilde{\omega}_{2}\right) / a\right]\right\}\right) .
\end{aligned}
$$

Assuming that $f_{1}$ or $\bar{f}_{2}$ is a massless particle, we can perform explicitly the integrals that appear in Eqs. (4.10) and (4.11). For this purpose, we make the change of variables $\left(\tilde{\omega}_{1}, \tilde{\omega}_{2}\right) \rightarrow(\rho, \zeta)$, where

$$
\rho \equiv \tilde{\omega}_{l} / \tilde{\omega}_{i}+1 \quad \text { and } \zeta \equiv \tilde{\omega}_{i}^{2} / m^{2}
$$

and here we label the massless and massive (with mass $m$ ) particles with $l$ and $i$ indices, respectively. Applying Eq. (4.12) in Eqs. (4.10) and (4.11) with $m_{l}=0$, we have

$$
\begin{aligned}
\Gamma^{p_{1} \rightarrow p_{2}=} & \frac{G_{\mathrm{eff}}^{2}\left(c_{V}^{2}+c_{A}^{2}\right) m^{6}}{2 \pi^{4} a e^{\pi \Delta M / a}} \int_{1}^{\infty} d \rho(\rho-1)^{2} \int_{1}^{\infty} d \zeta \zeta^{3 / 2}(\zeta-1)^{1 / 2} \\
& \times K_{2 i \Delta M / a}\left[2 m \rho \zeta^{1 / 2} / a\right] \\
\mathcal{W}_{\text {massive }}^{p_{1} \rightarrow p_{2}}= & \frac{G_{\mathrm{eff}}^{2}\left(c_{V}^{2}+c_{A}^{2}\right) m^{7}}{2 \pi^{4} a e^{\pi \Delta M / a}} \int_{1}^{\infty} d \rho(\rho-1)^{2} \int_{1}^{\infty} d \zeta \zeta^{2}(\zeta-1)^{1 / 2} \\
& \times K_{2 i \Delta M / a}\left[2 m \rho \zeta^{1 / 2} / a\right],
\end{aligned}
$$

and

$$
\begin{aligned}
\mathcal{W}_{\text {massless }}^{p_{1} \rightarrow p_{2}}= & \frac{G_{\mathrm{eff}}^{2}\left(c_{V}^{2}+c_{A}^{2}\right) m^{7}}{2 \pi^{4} a e^{\pi \Delta M / a}} \int_{1}^{\infty} d \rho(\rho-1)^{3} \int_{1}^{\infty} d \zeta \zeta^{2}(\zeta-1)^{1 / 2} \\
& \times K_{2 i \Delta M / a}\left[2 m \rho \zeta^{1 / 2} / a\right] .
\end{aligned}
$$

By using Eq. (6.592.4) of Ref. [13] to perform the $\zeta$ integration in Eqs. (4.13)-(4.15), we obtain

$$
\begin{aligned}
\Gamma^{p_{1} \rightarrow p_{2}=}= & \frac{G_{\mathrm{eff}}^{2}\left(c_{V}^{2}+c_{A}^{2}\right) m^{3} a^{2}}{8 \pi^{7 / 2} e^{\pi \Delta M / a}} \int_{1}^{\infty} d \rho\left(\rho^{-1}-2 \rho^{-2}+\rho^{-3}\right) \\
& \times G_{13}^{30}\left(\frac{m^{2} \rho^{2}}{a^{2}} \mid \begin{array}{l}
0 \\
-3 / 2,3 / 2+i \Delta M / a, 3 / 2-i \Delta M / a
\end{array}\right),
\end{aligned}
$$

$$
\begin{aligned}
\mathcal{W}_{\text {massive }}^{p_{1} \rightarrow p_{2}}= & \frac{G_{\mathrm{eff}}^{2}\left(c_{V}^{2}+c_{A}^{2}\right) m^{3} a^{3}}{8 \pi^{7 / 2} e^{\pi \Delta M / a}} \int_{1}^{\infty} d \rho\left(\rho^{-2}-2 \rho^{-3}+\rho^{-4}\right) \\
& \times G_{13}^{30}\left(\frac{m^{2} \rho^{2}}{a^{2}} \mid \begin{array}{ll}
0 & -3 / 2,2+i \Delta M / a, 2-i \Delta M / a
\end{array}\right),
\end{aligned}
$$

$$
\begin{aligned}
\mathcal{W}_{\text {massless }}^{p_{1} \rightarrow p_{2}}= & \frac{G_{\mathrm{eff}}^{2}\left(c_{V}^{2}+c_{A}^{2}\right) m^{3} a^{3}}{8 \pi^{7 / 2} e^{\pi \Delta M / a}} \\
& \times \int_{1}^{\infty} d \rho\left(\rho^{-1}-3 \rho^{-2}+3 \rho^{-3}-\rho^{-4}\right) \\
& \times G_{13}^{30}\left(\frac{m^{2} \rho^{2}}{a^{2}} \mid-3 / 2,2+i \Delta M / a, 2-i \Delta M / a\right),
\end{aligned}
$$

where $G_{p q}^{m n}\left(\left.x\right|_{b_{1}, \ldots, b_{q}} ^{a_{1}, \ldots, a_{p}}\right.$ ) are the Meijer's $G$ functions (see Ref. [13] for their definition and properties). Defining $v$ $\equiv \rho^{2}$ in Eqs. (4.16)-(4.18), and using Eq. (7.811.3) of Ref. [13], we can integrate these expressions. The Meijer's $G$ function sums that appear as a result can be simplified by using their properties. Eventually, we obtain

$$
\begin{aligned}
& \Gamma^{p_{1} \rightarrow p_{2}}=\frac{G_{\mathrm{eff}}^{2}\left(c_{V}^{2}+c_{A}^{2}\right) m^{3} a^{2}}{32 \pi^{7 / 2} e^{\pi \Delta M / a}} \\
& \times G_{24}^{40}\left(\frac{m^{2}}{a^{2}} \mid \begin{array}{l}
3 / 2,2 \\
1 / 2, \quad-3 / 2,3 / 2+i \Delta M / a, 3 / 2-i \Delta M / a
\end{array}\right),
\end{aligned}
$$

$$
\begin{aligned}
\mathcal{W}_{\text {massive }}^{p_{1} \rightarrow p_{2}} & =\frac{G_{\mathrm{eff}}^{2}\left(c_{V}^{2}+c_{A}^{2}\right) m^{3} a^{3}}{32 \pi^{7 / 2} e^{\pi \Delta M / a}} \\
& \times G_{35}^{50}\left(\frac{m^{2}}{a^{2}} \mid \begin{array}{ll}
0,2,5 / 2 \\
1 / 2,1,-3 / 2,2+i \Delta M / a, 2-i \Delta M / a
\end{array}\right),
\end{aligned}
$$

$$
\begin{aligned}
& \mathcal{W}_{\text {massless }}^{p_{1} \rightarrow p_{2}}=\frac{3 G_{\mathrm{eff}}^{2}\left(c_{V}^{2}+c_{A}^{2}\right) m^{3} a^{3}}{64 \pi^{7 / 2} e^{\pi \Delta M / a}} \\
& \times G_{24}^{40}\left(\frac{m^{2}}{a^{2}} \mid \begin{array}{l}
2,5 / 2 \\
1 / 2,-3 / 2,2+i \Delta M / a, 2-i \Delta M / a
\end{array}\right) .
\end{aligned}
$$


In the case where both $f_{1}$ and $\bar{f}_{2}$ are massless particles, this is more convenient to obtain the total transition rate by first integrating in momenta $\widetilde{\mathbf{k}}_{1}$ and $\widetilde{\mathbf{k}}_{2}$. Thus, we first write [see Eq. (4.6)]

$$
\begin{aligned}
\Gamma^{p_{1} \rightarrow p_{2}=}= & \frac{G_{\mathrm{eff}}^{2}\left(c_{V}^{2}+c_{A}^{2}\right)}{2 \pi^{4}} \int_{-\infty}^{+\infty} d \xi e^{2 i \Delta M \xi} \\
& \times\left\{\int_{0}^{\infty} d \tilde{\omega} \tilde{\omega}^{2} \exp \left[\frac{2 i \tilde{\omega}}{a}(\sinh a \xi+i \epsilon)\right]\right\}^{2},
\end{aligned}
$$

where $\epsilon>0$ is a regulator that ensures the convergence of the frequency integral above. The corresponding total emitted power is

$$
\begin{aligned}
\mathcal{W}^{p_{1} \rightarrow p_{2}=}= & \frac{G_{\mathrm{eff}}^{2}\left(c_{V}^{2}+c_{A}^{2}\right)}{\pi^{4}} \int_{-\infty}^{+\infty} d \xi e^{2 i \Delta M \xi} \int_{0}^{\infty} d \tilde{\omega}_{1} \tilde{\omega}_{1}^{3} \\
& \times \exp \left[\frac{2 i \tilde{\omega}_{1}}{a}(\sinh a \xi+i \epsilon)\right] \int_{0}^{\infty} d \tilde{\omega}_{2} \tilde{\omega}_{2}^{2} \\
& \times \exp \left[\frac{2 i \tilde{\omega}_{2}}{a}(\sinh a \xi+i \epsilon)\right] .
\end{aligned}
$$

By performing the frequency integrals and defining the new variable $w \equiv e^{a \xi}$, Eqs. (4.22), (4.23) become

$$
\begin{aligned}
\Gamma^{p_{1} \rightarrow p_{2}} & =-\frac{2 G_{\mathrm{eff}}^{2}\left(c_{V}^{2}+c_{A}^{2}\right) a^{5}}{\pi^{4}} \int_{0}^{\infty} d w \frac{w^{5+2 i \Delta M / a}}{\left(w^{2}-1+2 i \epsilon w\right)^{6}}, \\
\mathcal{W}^{p_{1} \rightarrow p_{2}} & =-\frac{12 i G_{\mathrm{eff}}^{2}\left(c_{V}^{2}+c_{A}^{2}\right) a^{6}}{\pi^{4}} \int_{0}^{\infty} d w \frac{w^{6+2 i \Delta M / a}}{\left(w^{2}-1+2 i \epsilon w\right)^{7}} .
\end{aligned}
$$

Solving the integrals that appear in Eqs. (4.24) and (4.25) (see the Appendix), we obtain

$$
\Gamma^{p_{1} \rightarrow p_{2}}=\frac{G_{\mathrm{eff}}^{2}\left(c_{V}^{2}+c_{A}^{2}\right)}{60 \pi^{3}}\left(\frac{4 a^{4} \Delta M+5 a^{2} \Delta M^{3}+\Delta M^{5}}{e^{2 \pi \Delta M / a}-1}\right)
$$

and

$\mathcal{W}^{p_{1} \rightarrow p_{2}}$

$$
\begin{aligned}
= & \frac{G_{\mathrm{eff}}^{2}\left(c_{V}^{2}+c_{A}^{2}\right)}{3840 \pi^{3}} \\
& \times\left(\frac{225 a^{6}+1036 a^{4} \Delta M^{2}+560 a^{2} \Delta M^{4}+64 \Delta M^{6}}{e^{2 \pi \Delta M / a}+1}\right) .
\end{aligned}
$$

In the next sections we use these formulas to investigate some selected reactions.

\section{ACCELERATED PROTON AND NEUTRON DECAY}

Let us now consider the processes

$$
p \rightarrow n e^{+} \nu_{e}
$$

and

$$
n \rightarrow p e^{-\bar{\nu}_{e}}
$$

for uniformly accelerated protons and neutrons, respectively. We will assume the neutrino mass to vanish because even if this is not so, it would be neglectable in comparison with any other energy scale involved in the problem. The effective coupling constant $G_{\text {eff }}=G_{p n}$ for processes (5.1), (5.2) is obtained by imposing that the mean proper lifetime of inertial neutrons is $887 \mathrm{~s}$ [14], i.e.,

$$
\Gamma_{i n}^{n \rightarrow p} \equiv \Gamma^{n \rightarrow p}(a \rightarrow 0)=1 / 887 \mathrm{~s}^{-1} .
$$

This phenomenological procedure has the advantage of by passing any uncertainties on the influence of the nucleon inner structure. For sake of convenience, we take the $a \rightarrow 0$ limit in Eq. (4.6) rather than in Eq. (4.19), obtaining

$$
\begin{aligned}
\frac{d \Gamma_{i n}^{n \rightarrow p}}{d^{3} \widetilde{\mathbf{k}}_{e} d^{3} \widetilde{\mathbf{k}}_{\nu}}= & \frac{4 G_{p n}^{2}}{(2 \pi)^{6} \tilde{\omega}_{e} \tilde{\omega}_{\nu}} \int_{-\infty}^{+\infty} d \xi e^{2 i \xi\left(\Delta M+\tilde{\omega}_{e}+\tilde{\omega}_{\nu}\right)} \\
& \times\left(\tilde{\omega}_{e} \tilde{\omega}_{\nu}+\widetilde{\mathbf{k}}_{e} \cdot \widetilde{\mathbf{k}}_{\nu}\right) \\
= & \frac{2 G_{p n}^{2}}{(2 \pi)^{5}}\left(1+\frac{\tilde{\mathbf{k}}_{e} \cdot \tilde{\mathbf{k}}_{\nu}}{\tilde{\omega}_{e} \tilde{\omega}_{\nu}}\right) \delta\left(\tilde{\omega}_{e}+\tilde{\omega}_{\nu}-\Delta M\right),
\end{aligned}
$$

where we have used $c_{V}=c_{A}=1$ [15] since only left-handed massless neutrinos are known to exist. After integrating Eq. (5.4) in angular coordinates and in $\widetilde{\omega}_{e}$, we find

$$
\Gamma_{i n}^{n \rightarrow p}=\frac{G_{p n}^{2}}{\pi^{3}} \int_{0}^{\Delta M-m_{e}} d \tilde{\omega}_{\nu} \tilde{\omega}_{\nu}^{2}\left(\Delta M-\tilde{\omega}_{\nu}\right) \sqrt{\left(\Delta M-\tilde{\omega}_{\nu}\right)^{2}-m_{e}^{2}} .
$$

Evaluating numerically Eq. (5.5) with $m_{e}=0.511 \mathrm{MeV}$, and $\Delta M=\left(m_{n}-m_{p}\right)=1.29 \mathrm{MeV}$, we end up with $\Gamma_{i n}^{n \rightarrow p}=1.81$ $\times 10^{-3} G_{p n}^{2} \mathrm{MeV}^{5}$. Hence by imposing condition (5.3), we obtain $G_{p n}=1.74 G_{F}$, where $G_{F} \equiv 1.166 \times 10^{-5} \mathrm{GeV}^{-2}$ is the Fermi coupling constant [14]. Now we are able to use Eq. (4.19) to plot in Fig. 1 the proton and neutron mean proper lifetimes $\tau_{p}(a)=\left(\Gamma^{p \rightarrow n}\right)^{-1}$ and $\tau_{n}(a)=\left(\Gamma^{n \rightarrow p}\right)^{-1}$, respectively. Let us note that

$$
\tau_{n}(a)=e^{-2 \pi|\Delta M| / a} \tau_{p}(a) .
$$

We have only considered accelerations $a \ll m_{p}=938 \mathrm{MeV}$ in order to respect our no-recoil condition (see Sec. II). We call attention to the fact that for accelerations $a \gg a_{c} \equiv 2 \pi|\Delta M|$ $\approx 8 \mathrm{MeV}$, we have $\tau_{p}(a) \approx \tau_{n}(a)$. This is easier to understand in the coaccelerated frame with the current, where (according to the FDU effect $[5,6]$ ) a thermal bath of Rindler particles with temperature $T_{\mathrm{FDU}}=a / 2 \pi$ is "attached" to the 


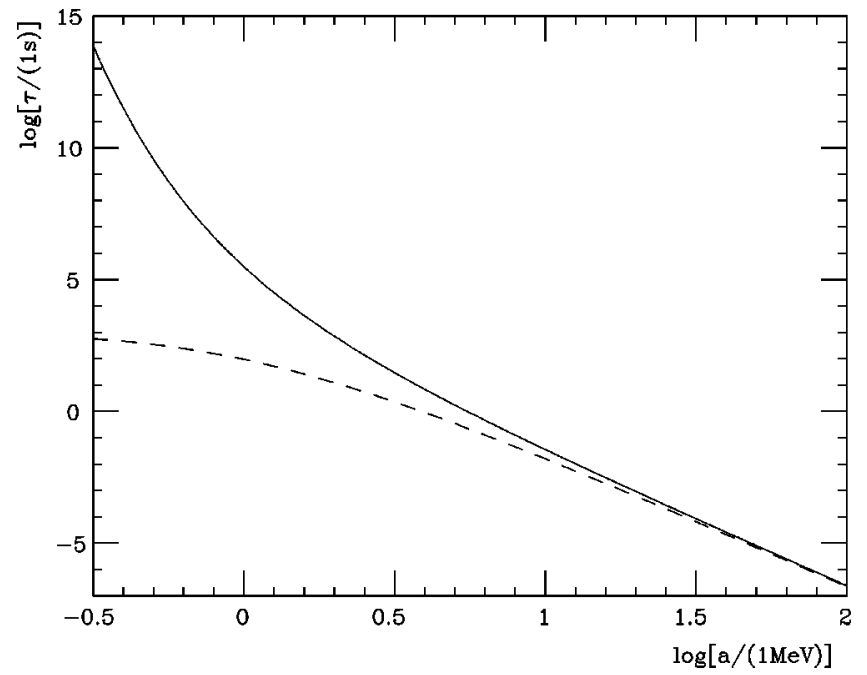

FIG. 1. The mean proper lifetime of protons, $\tau_{p}$ (full line), and neutrons, $\tau_{n}$ (dashed line), are plotted as functions of their proper acceleration $a$. Note that $\tau_{p} \rightarrow+\infty$ and $\tau_{n} \rightarrow 887 \mathrm{~s}$ as $a \rightarrow 0$. For accelerations $a \gg a_{c} \equiv 2 \pi \Delta M \approx 8 \mathrm{MeV}$ we have that $\tau_{p} \approx \tau_{n}$.

current. Thus, for $a \gg a_{c}$ we have $T_{\mathrm{FDU}} \gg|\Delta M|$, which leads both nucleons to behave similarly. (See Ref. [7] for a more comprehensive discussion on this issue.)

In order to estimate how much energy is carried out in form of leptons, we may use Eqs. (4.20) and (4.21) to obtain $\mathcal{W}_{j}^{p \rightarrow n}$ and $\mathcal{W}_{j}^{n \rightarrow p}=e^{2 \pi|\Delta M| / a} \mathcal{W}_{j}^{p \rightarrow n}$ for $j=e, \nu$. Although $\mathcal{W}_{e}^{p \rightarrow n}$ and $\mathcal{W}_{\nu}^{p \rightarrow n}$ (as well as $\mathcal{W}_{e}^{n \rightarrow p}$ and $\mathcal{W}_{\nu}^{n \rightarrow p}$ ) are not manifestly identical, they seem to be according to Fig. 2.

In order to investigate the energy distribution of the emitted leptons, let us define the normalized energy distribution

$$
\mathcal{N}_{j}^{p_{1} \rightarrow p_{2}} \equiv \frac{1}{\Gamma^{p_{1} \rightarrow p_{2}}} \frac{d \Gamma^{p_{1} \rightarrow p_{2}}}{d \tilde{\omega}_{j}}
$$

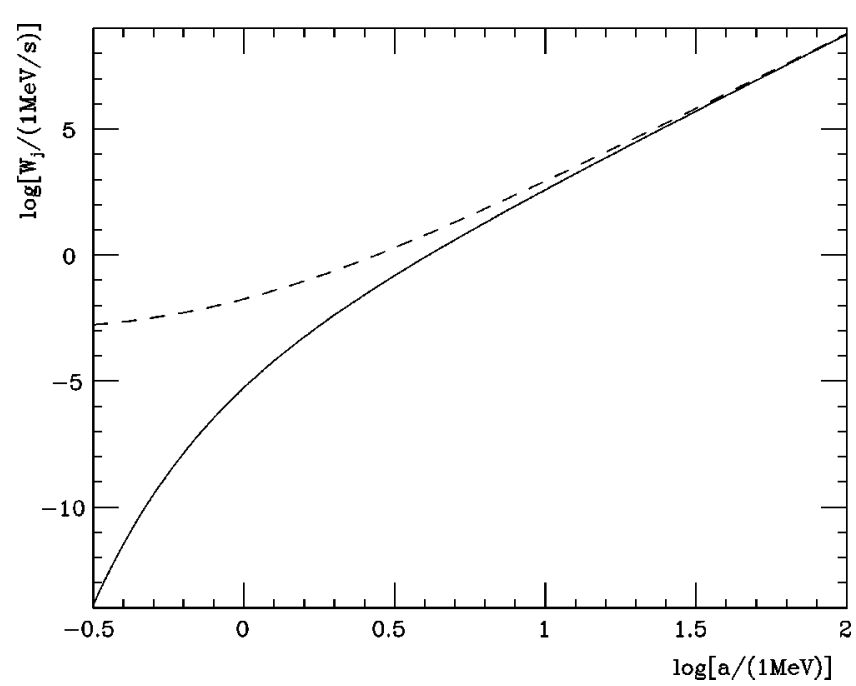

FIG. 2. $\mathcal{W}_{j}^{p \rightarrow n}$ and $\mathcal{W}_{j}^{n \rightarrow p}$ are plotted in full and dashed lines, respectively, for $j=e, \nu$, as functions of the nucleon proper accelerations. Our numerical results suggest that $\mathcal{W}_{e}^{p \rightarrow n}=\mathcal{W}_{\nu}^{p \rightarrow n}$ and $\mathcal{W}_{e}^{n \rightarrow p}=\mathcal{W}_{\nu}^{n \rightarrow p}$.

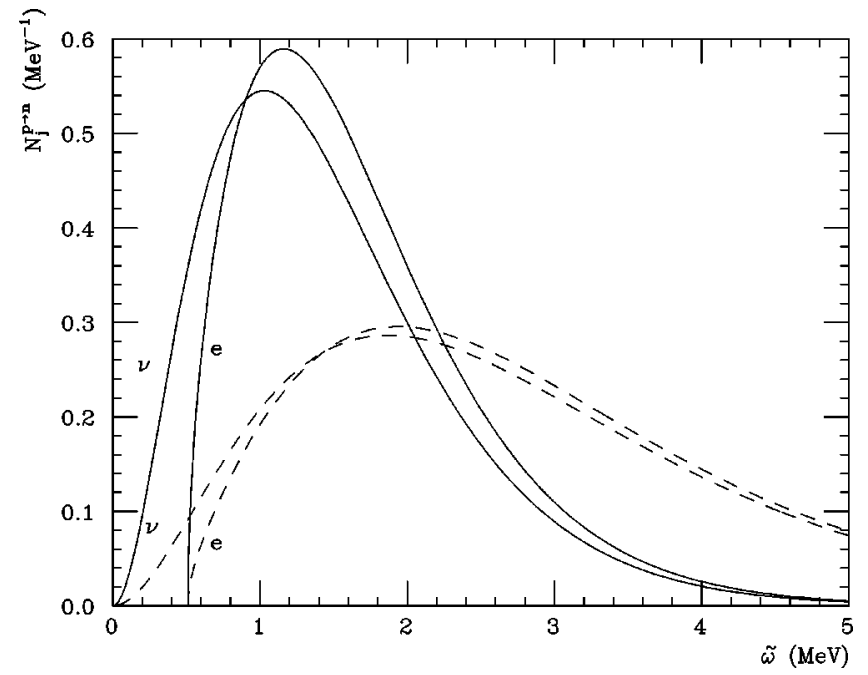

FIG. 3. The normalized energy distribution of emitted positrons, $\mathcal{N}_{e}^{p \rightarrow n}$, and neutrinos, $\mathcal{N}_{\nu}^{p \rightarrow n}$, are plotted for two values of the proton's proper acceleration: $a=1.0 \mathrm{MeV}$ (full line) and $2.0 \mathrm{MeV}$ (dashed line). Note that the typical energy of the emitted particles (in the inertial frame instantaneously at rest with the proton) is given by $\tilde{\omega} \approx a$.

with $j=e, \nu$, where $d \Gamma^{p_{1} \rightarrow p_{2}} / d \tilde{\omega}_{j}$ is defined in Eq. (4.9). Note that $\mathcal{N}_{j}^{p \rightarrow n}=\mathcal{N}_{j}^{n \rightarrow p}$. In Fig. 3 we plot the distributions $\mathcal{N}_{j}^{p \rightarrow n}$ for two values of acceleration: $a=1.0$ and $2.0 \mathrm{MeV}$. We see that the typical energy (in the inertial frame instantaneously at rest with the nucleon) of the emitted electrons and neutrinos is $\tilde{\omega} \approx a$, which justifies our no-recoil condition.

In order to roughly estimate how small is the proper lifetime of circularly moving protons at the CERN Large Hadron Collider (LHC) we use directly Eq. (5.6) with $a=a_{\mathrm{LHC}}$ $\approx 10^{-8} \mathrm{MeV}$ for the proton's proper acceleration, obtaining $\tau_{p}\left(a_{\text {LHC }}\right) \approx 10^{3 \times 10^{8}} \mathrm{yr}$, where we have used that $\tau_{n}(a$ $\left.\ll m_{e},|\Delta M|\right) \approx 10^{3}$ s. Although Eq. (5.6) was derived assuming uniformly accelerated motion, this should not be seen as a major problem: Because of the huge proper lifetime obtained for the proton, our estimation turns out to be nonsensitive up to an inaccuracy of hundreds of thousands of orders of magnitude (which should not be the case).

Astrophysics seems to provide much more suitable conditions for the observation of the decay of accelerated protons. Although our decay rate (4.19) was obtained considering uniformly accelerated protons, let us assume that this is approximately valid for circularly moving protons with proper acceleration $a \gg \Delta M, 1 / R$, where $R$ is the local curvature radius of the proton trajectory. Indeed we can test this assumption, e.g., for two-level scalar systems, whose excitation rates, at the tree level, are given by [16]

$$
\Gamma_{\operatorname{lin}}=\frac{c_{0}^{2}}{2 \pi} \frac{\Delta E}{e^{2 \pi \Delta E / a}-1}
$$

and 


$$
\Gamma_{\text {cir }}=\frac{c_{0}^{2}}{2 \pi} \frac{a e^{-\sqrt{12} \Delta E / a}}{2 \sqrt{12}}
$$

for uniformly accelerated and circularly moving relativistic sources, respectively, where $c_{0}$ is a small coupling constant and $\Delta E$ is the two-level system energy gap. Note that in the limit $a \gg \Delta E$, Eqs. (5.8) and (5.9) give us $\Gamma_{\text {lin }} / \Gamma_{\text {cir }}=1.103$.

In order to illustrate an astrophysical situation where process (5.1) may be important, let us consider a cosmic ray proton with energy $E_{p}=\gamma m_{p} \approx 1.6 \times 10^{14} \mathrm{eV}$ under the influence of a magnetic field $B \approx 10^{14} \mathrm{G}$ of a typical pulsar. Protons under these conditions have proper accelerations of $a_{B}$ $=\gamma e B / m_{p} \approx 110 \mathrm{MeV} \gg|\Delta M|$. For practical purposes the acceleration of the proton will be assumed as constant along the process. For the chosen values of $E_{p}$ and $B$, the proton is confined in a cylinder with typical radius $R \approx \gamma^{2} / a_{B} \approx 5$ $\times 10^{-3} \mathrm{~cm} \ll l_{B}$, where $l_{B}$ is the typical size of the magnetic field region. According to Eq. (4.19) we obtain $\tau_{p} \approx 10^{-7} \mathrm{~s}$. As a result, protons would have a "laboratory" mean lifetime of $t_{p}=\gamma \tau_{p} \approx 10^{-1} \mathrm{~s}$. For $l_{B} \approx 10^{7} \mathrm{~cm}$, we obtain that less than $\left|\Delta N_{p} / N_{p}\right|=\left(1-e^{-l_{B} / t_{p}}\right) \approx l_{B} / t_{p} \approx 1 \%$ of the protons would decay via process (5.1). We note that we did not take into account the influence of the magnetic field on the emitted positron. Clearly a more precise estimation should take into account this effect as well as other ones as, e.g., the nonuniformity of the magnetic field and energy losses through electromagnetic sinchrotron radiation. The last one in particular may not be a problem since energy may be furnished to the proton from dynamo processes. A more careful analysis of such astrophysical issues would be welcome but this is beyond the scope of the present fieldtheoretical investigation.

\section{NEUTRINO EMISSION FROM UNIFORMLY ACCELERATED ELECTRONS}

In this section, we will consider the emission of neutrinos from accelerated electrons:

$$
e^{-} \rightarrow e^{-} \nu_{e} \bar{\nu}_{e}
$$

The description of the creation of neutrino-antineutrino pairs by electrons in an external electromagnetic field in the context of the standard model is contained in Sec. 6.1 of Ref. [2]. Here we analyze this process for uniformly accelerated electrons by using the formulas derived in Sec. IV where both emitted fermions are massless. From Eqs. (4.26) and (4.27) we get for the emission rate of $\nu_{e} \bar{\nu}_{e}$ pairs

$$
\Gamma_{\nu \bar{\nu}}=\frac{G_{e \nu}^{2} a^{5}}{15 \pi^{4}},
$$

and for the total radiated power

$$
\mathcal{W}_{\nu \nu}=\frac{15 G_{e \nu}^{2} a^{6}}{256 \pi^{3}},
$$

where we have used $\Delta M=0, c_{V}=c_{A}=1$ and $G_{e \nu}$ is the corresponding effective coupling constant.

In order to determine the value of $G_{e \nu}$, we assume that Eq. (6.3) describes the instantaneous emitted power from an electron with arbitrary world line at the point where it has proper acceleration $a$. This is indeed verified for photon (see Larmor formula in Ref. [17]) and scalar particle [18] emission from accelerated sources. [We emphasize that this equivalence is not fully (although it is approximately) verified for Eq. (6.2), which depends in general on the source's world line.] Thus we will impose that Eq. (6.3) gives the radiated power for the neutrino emission from circularly moving relativistic electrons in a uniform magnetic field $B$ provided that $a=\gamma e B / m_{e} \ll m_{e}$ (no-recoil condition). Here $\gamma$ is the usual Lorentz factor for the electron and $e$ is its electric charge. The differential emission rate of $\nu_{e} \bar{\nu}_{e}$ pairs in a background magnetic field was calculated in detail [2] (see Ref. [8] for the form used below):

$$
\begin{aligned}
\frac{d \Gamma_{\nu \bar{\nu}}^{L P}}{d s}= & \frac{G_{F}^{2} m_{e}^{4}}{16(2 \pi)^{3}} \frac{m_{e}}{\gamma} \frac{\chi^{5} s^{3+1 / 2}}{\left(1+\chi s^{3 / 2}\right)^{4}} \times\left\{\left(C_{V}^{2}+C_{A}^{2}\right) \frac{\chi^{2} s^{3}}{\left(1+\chi s^{3 / 2}\right)}\right. \\
& \times \int_{s}^{\infty}\left[2+\frac{1}{3}(2 s+y)(y-s)^{2}\right] \operatorname{Ai}(y) d y+\left(C_{V}^{2}+C_{A}^{2}\right) \\
& \times\left[\int_{s}^{\infty}\left[6+(y-s)\left(s^{2}+(s-y)^{2}\right)\right] \operatorname{Ai}(y) d y-s \operatorname{Ai}(s)\right] \\
& \left.+8 s C_{A}^{2}\left[\frac{3}{4}\left(\int_{s}^{\infty}(s-y)^{2} \operatorname{Ai}(y) d y\right)+\operatorname{Ai}(s)\right]\right\}, \quad \text { (6.4) }
\end{aligned}
$$

where $\chi \equiv a / m_{e}, \operatorname{Ai}(z)$ is the Airy function, and $s$ $\in[0, \gamma / \chi]$ is defined such that

$$
\omega_{\nu}+\omega_{\nu}^{-} \equiv \frac{m_{e} \gamma \chi s^{3 / 2}}{\left(1+\chi s^{3 / 2}\right)}
$$

The parameters $C_{V}$ and $C_{A}$ give the vector and axial contributions to the electric current, respectively. Using Eqs. (6.4) and (6.5) we have, in the limit $\chi \ll 1$,

$$
\mathcal{W}_{\nu \bar{\nu}}^{L P}=\int_{0}^{\gamma / \chi} d s\left(\omega_{\nu}+\omega_{\nu}^{-}\right) \frac{d \Gamma_{\nu \bar{\nu}}^{L P}}{d s}=\frac{5\left(2 C_{V}^{2}+23 C_{A}^{2}\right)}{108 \pi^{3}} G_{F}^{2} m_{e}^{6} \chi^{6} .
$$

Letting $C_{V}^{2}=0.93$ and $C_{A}^{2}=0.25$ [9], we have $\mathcal{W}_{\nu \bar{\nu}}^{L P}=1.14$ $\times 10^{-2} G_{F}^{2} a^{6}$. By comparing this expression with our Eq. (6.3) we obtain $G_{e \nu}=2.45 G_{F}$. In Figs. 4 and 5 we plot Eqs. (6.2) and (6.3), respectively, for uniformly accelerated electrons with $a \leqslant m_{e}$.

The normalized energy distribution of emitted neutrinoantineutrino

$$
\mathcal{N}_{\nu \bar{\nu}} \equiv \frac{1}{\Gamma_{\nu \bar{\nu}}} \frac{d \Gamma_{\nu \bar{\nu}}}{d \tilde{\omega}_{\nu}}
$$




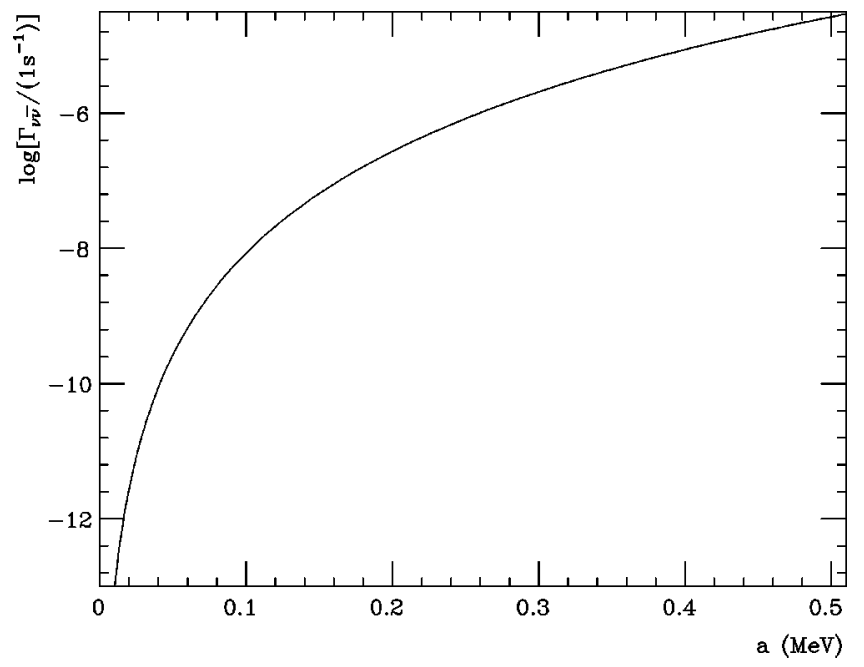

FIG. 4. The emission probability per proper time of $\nu_{e} \bar{\nu}_{e}$ pairs is plotted for $a \leqslant m_{e}$.

is plotted in Fig. 6 for electrons with proper acceleration $a$ $=0.1 \mathrm{MeV}$ and $0.2 \mathrm{MeV}$, where [see Eq. (4.9)]

$$
\frac{d \Gamma_{\nu \bar{\nu}}}{d \tilde{\omega}_{\nu}}=\frac{2 G_{e \nu}^{2}}{\pi^{4} a} \tilde{\omega}_{\nu}^{2} \int_{0}^{\infty} d \tilde{\omega}_{\nu}^{-} \tilde{\omega}_{\nu}^{2} K_{0}\left[2\left(\tilde{\omega}_{\nu}+\tilde{\omega}_{\nu}^{-}\right) / a\right]
$$

(Neutrinos and antineutrinos have identical emission energy distribution.) Note again that $a$ defines the typical energy of the emitted neutrinos.

\section{DISCUSSIONS}

We have investigated the weak interaction emission of spin-1/2 fermions from classical and semiclassical currents. As a particular application of modeling the accelerated particle by a semiclassical current, we have analyzed the inverse $\beta$ decay of uniformly accelerated protons. We have shown that although protons in laboratory storage rings are not

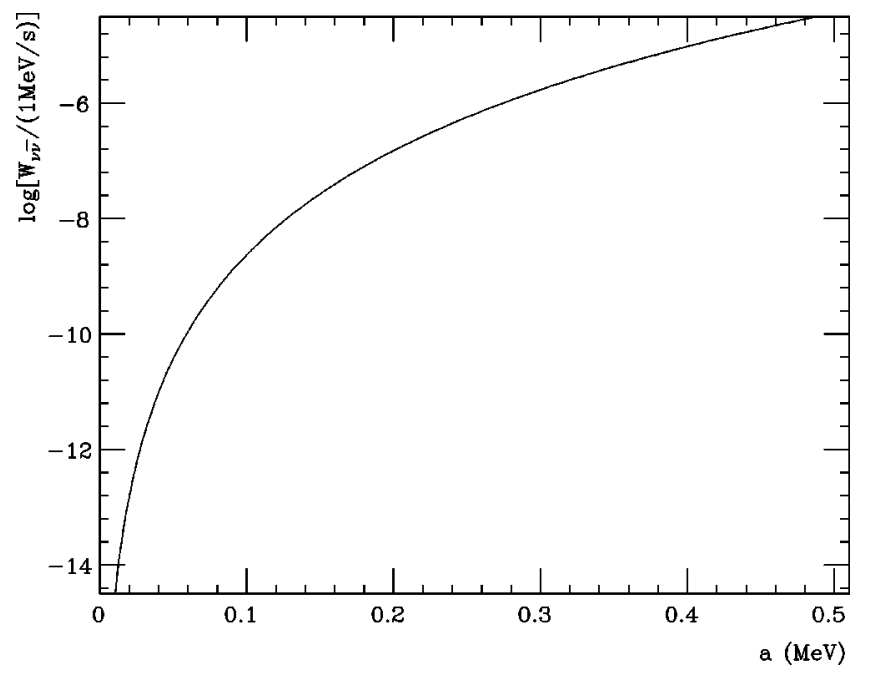

FIG. 5. The total radiated power in form of neutrinos is plotted for $a \leqslant m_{e}$.

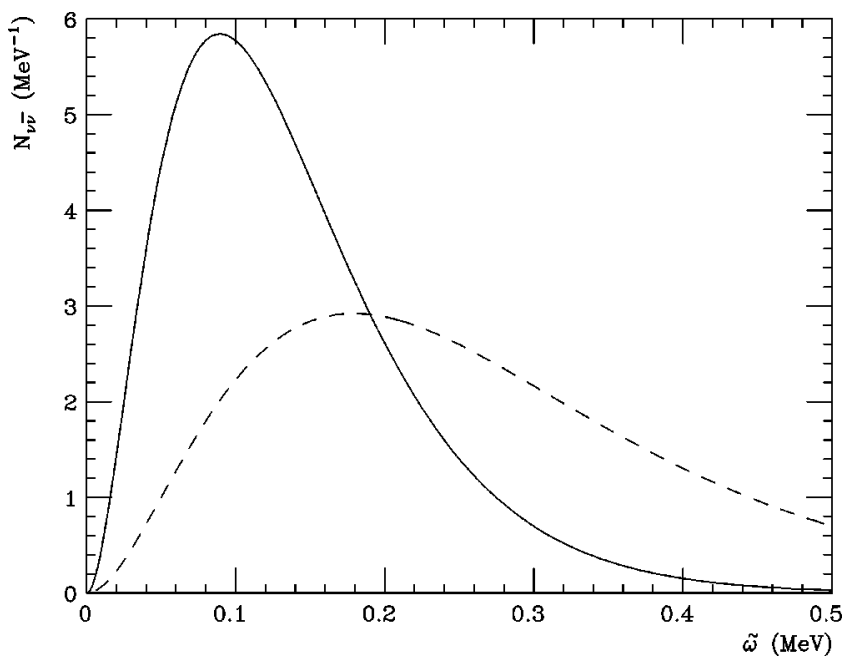

FIG. 6. The normalized energy distribution of the emitted neutrinos (and antineutrinos) is plotted for two values of the electron's proper acceleration: $a=0.1 \mathrm{MeV}$ (full line) and $0.2 \mathrm{MeV}$ (dashed line). Note that the typical energy of the emitted particles (in the inertial frame instantaneously at rest with the electron) is given by $\tilde{\omega} \approx a$.

likely to decay in this way, under some astrophysical conditions high-energy protons in background magnetic fields may have a considerably short lifetime. Moreover, we have analyzed the modification of the usual $\beta$ decay for uniformly accelerated neutrons. This may be of some relevance when neutrons are under the influence of strong background gravitational fields. Although a full curved spacetime calculation is desirable to treat these situations, our calculation should be a good approximation when the gravitational field is " $m o d-$ erate" [18]. In this case, neutrons can be treated as being accelerated in Minkowski space.

By restricting our semiclassical current to behave classically, we were able to use our formalism to investigate the neutrino-antineutrino pair emission from uniformly accelerated electrons and compare our results with the ones in the literature obtained by quantizing the electron field in a background magnetic field. Our formalism allows the utilization of currents associated with more general world lines. Depending on the accuracy level required, however, one can use directly the formulas derived for uniformly accelerated currents. This may be particularly useful in some astrophysical situations.

\section{ACKNOWLEDGMENTS}

The authors are thankful to J. C. Montero, V. Pleitez, and A. A. Natale for discussions. D. V. was fully supported by Fundação de Amparo à Pesquisa do Estado de São Paulo while G.M. was partially supported by Conselho Nacional de Desenvolvimento Científico e Tecnológico.

\section{APPENDIX: INTEGRATION OF EQS. (4.24), (4.25)}

In order to solve Eqs. (4.24) and (4.25) let us consider the integral 


$$
\mathcal{I}_{n}^{+} \equiv \int_{0}^{\infty} d w \frac{w^{n+2 i \Delta M / a}}{\left(w^{2}-1+2 i \epsilon w\right)^{n+1}}, \quad n \in \mathrm{N}
$$

Note that the analytic extension of the integrand above has poles of order $(n+1)$ at $w^{ \pm}= \pm 1-i \epsilon+\mathcal{O}\left(\epsilon^{2}\right)$. This implies that we can make $\epsilon=0$ in Eq. (A1) provided we contour the pole at $w^{+}=1$ by the upper half-plane, i.e.,

$$
\mathcal{I}_{n}^{+}=\int_{\gamma_{+}} d w \frac{w^{n+2 i \Delta M / a}}{\left(w^{2}-1\right)^{n+1}},
$$

where $\gamma_{ \pm} \equiv\left[0,1-\epsilon^{\prime}\right] \cup\left\{1 \pm \epsilon^{\prime} e^{i \theta} ; \quad \theta \in[0, \pi]\right\} \cup\left[1+\epsilon^{\prime}, \infty\right)$ with $\epsilon^{\prime} \rightarrow 0_{+}$. Using the residue theorem we see that

$$
\mathcal{I}_{n}^{-}-\mathcal{I}_{n}^{+}=2 \pi i \operatorname{Res}\left(f_{n}\right)_{w=1},
$$

where $\mathcal{I}_{n}^{-}$is obtained substituting $\gamma_{+}$by $\gamma_{-}$in Eq. (A2), and we denote the residue value of the function

$$
f_{n}(w) \equiv \frac{w^{n+2 i \Delta M / a}}{\left(w^{2}-1\right)^{n+1}}
$$

at the point $w=w^{ \pm}$by $\operatorname{Res}\left(f_{n}\right)_{w=w^{ \pm}}$. Now, let us define

$$
\mathcal{I}_{n} \equiv \int_{-\infty}^{+\infty} d w \frac{w^{n+2 i \Delta M / a}}{\left(w^{2}-1+2 i \epsilon w\right)^{n+1}}
$$

which can be written for $\epsilon \rightarrow 0$ as

$$
\mathcal{I}_{n}=(-1)^{n} e^{-2 \pi \Delta M / a} \mathcal{I}_{n}^{-}+\mathcal{I}_{n}^{+} .
$$

Since the integrand of $\mathcal{I}_{n}$ is analytic in the upper half-plane and goes to zero as $|w|^{-(n+2)}$ as $|w| \rightarrow \infty$, it follows that $\mathcal{I}_{n}$ $=0$. As a consequence Eqs. (A6) and (A3) imply

$$
\mathcal{I}_{n}^{+}=\frac{-2 \pi i \operatorname{Res}\left(f_{n}\right)_{w=1}}{1+(-1)^{n} e^{2 \pi \Delta M / a}}
$$

with

$$
\operatorname{Res}\left(f_{n}\right)_{w=w^{ \pm}}=\left.\frac{1}{n !} \frac{d^{n}}{d w^{n}}\left\{\left(w-w^{ \pm}\right)^{n+1} f_{n}(w)\right\}\right|_{w=w^{ \pm}} .
$$

Using function (A4) to explicitly evaluate Eq. (A8) for $n$ $=5,6$, we obtain

$$
\mathcal{I}_{5}^{+}=\frac{-\pi}{120 a^{5}}\left(\frac{4 a^{4} \Delta M+5 a^{2} \Delta M^{3}+\Delta M^{5}}{e^{2 \pi \Delta M / a}-1}\right),
$$

and

$$
\begin{aligned}
\mathcal{I}_{6}^{+}= & \frac{i \pi}{46080 a^{6}} \\
& \times\left(\frac{225 a^{6}+1036 a^{4} \Delta M^{2}+560 a^{2} \Delta M^{4}+64 \Delta M^{6}}{e^{2 \pi \Delta M / a}+1}\right) .
\end{aligned}
$$

[1] V. B. Berestetskii, E. M. Lifshitz, and L. P. Pitaevskii, Quantum Electrodynamics (Butterworth-Heinemann, Oxford, 1982).

[2] V. N. Baier, V. M. Katkov, and V. M. Strakhovenko, Electromagnetic Processes at High Energies in Oriented Single Crystals (World Scientific, Singapore, 1998).

[3] V. L. Ginzburg and S. I. Syrovatskii, Usp. Fiz. Nauk 87, 65 (1965).

[4] R. Muller, Phys. Rev. D 56, 953 (1997).

[5] S. A. Fulling, Phys. Rev. D 7, 2850 (1973); P. C. W. Davies, J. Phys. A 8, 609 (1975).

[6] W. G. Unruh, Phys. Rev. D 14, 870 (1976).

[7] G. E. A. Matsas and D. A. T. Vanzella, Phys. Rev. D 59, 094004 (1999).

[8] L. B. Leinson and A. Pérez, Phys. Rev. D 59, 043002 (1999).

[9] A. D. Kaminker, K. P. Levenfish, D. G. Yakovlev, P. Amsterdamski, and P. Haensel, Phys. Rev. D 46, 3256 (1992).

[10] V. N. Baier and V. M. Katkov, Dokl. Akad. Nauk. SSSR 171, 313 (1966) [Sov. Phys. Dokl. 11, 947 (1967)]; J. D. Landstreet, Phys. Rev. 153, 1372 (1967); A. I. Nikishov and V. I. Ritus, Sov. Phys. JETP 67, 1313 (1988); A. D. Kaminker, K.
P. Levenfish, and D. G. Yakovlev, Sov. Astron. Lett. 17, 450 (1991); A. Vidaurre, A. Pérez, H. Sivak, J. Bernabéu, and J. M. Ibáñez, Astrophys. J. 448, 264 (1995).

[11] N. D. Birrell and P. C. W. Davies, Quantum Field Theory in Curved Spacetime (Cambridge University Press, Cambridge, England, 1982).

[12] C. Itzykson and J.-B. Zuber, Quantum Field Theory (McGrawHill, New York, 1980).

[13] I. S. Gradshteyn and I. M. Ryzhik, Table of Integrals, Series and Products (Academic, New York, 1980).

[14] Particle Data Group, C. Caso et al., Eur. Phys. J. C 3, 1 (1998).

[15] C. Quigg, Gauge Theory of the Strong, Weak, and Electromagnetic Interactions (Benjamin-Cummings, Reading, MA 1983).

[16] S. Takagi, Prog. Theor. Phys. 88, 1 (1986).

[17] J. D. Jackson, Classical Electrodynamics, 2nd ed. (Wiley, New York, 1975).

[18] L. C. B. Crispino, A. Higuchi, and G. E. A. Matsas, Class. Quantum Grav. 17, 19 (2000). 